\title{
IMPLANTE INMEDIATO Y PRESERVACIÓN DEL REBORDE CON LA TÉCNICA DE EXTRACCIÓN PARCIAL: SOCKET SHIELD
}

\author{
IMMEDIATE IMPLANT AND \\ RIDGE PRESERVATION WITH \\ THE PARTIAL EXTRACTION \\ TECHNIQUE: SOCKET SHIELD
}

\section{Britto Ebert Falcón Guerrero'}

1. Cirujano dentista. Doctor en estomatología. Especialista en Periodoncia e Implantología.

\section{RESUMEN}

Se presenta un caso clínico con un seguimiento de un año, donde se describe el procedimiento de implante inmediato y preservación del reborde con la técnica de extracción parcial: socket shield. Esta técnica consiste en conservar una porción de la raíz con su ligamento periodontal, permitiendo la instalación y función inmediata de un implante. Los resultados muestran la preservación de la cortical vestibular y el perfil de emergencia. Se observa también que es una técnica predecible, que acorta el tiempo de tratamiento y que obtiene un buen resultado tanto estético como funcional.

Palabras clave: Alveolo, escudo, extracción parcial, implante inmediato, socket shield.

\begin{abstract}
A clinical case with a one year follow-up is presented, where it is described the immediate implant and ridge preservation with the partial extraction technique: socket shield. This technique consists in preserving a portion of the root with its periodontal ligament, allowing the immediate installation and function of an implant. The results show the preservation of the buccal cortical and the emergence profile. It is also observed that it is a predictable technique, which shortens the treatment time and obtains a good result both aesthetic and functional.

Keywords: Immediate implant, socket shield, dental, partial extraction.
\end{abstract}




\section{INTRODUCCIÓN}

La instalación de implantes posterior a la extracción dental con carga inmediata es una alternativa viable para conservar los tejidos duros y blandos, previniendo la reabsorción ósea $(1,2)$. Sin embargo, tanto Araujo como Van Kesteren -mencionados por Al-Shabeeb (3)reportaron que la instalación de un implante en un alvéolo fresco, no previene el remodelado óseo que se da en las paredes del alvéolo. Del mismo modo, Kan y col. (4) mencionan que una desventaja en el tiempo de este procedimiento es la recesión y pérdida ósea de la cortical vestibular.

Salama y col. (5) recomendaron que mantener una raíz puede preservar el tejido circundante en el sitio del póntico. Asimismo, Hürzeler y col. (6) introducen la técnica del escudo o socket shield. Esta técnica mantiene intencionalmente un fragmento de raíz alrededor de un implante colocado inmediatamente, a fin de evitar alteraciones tisulares después de la extracción del diente. Posteriormente, se han realizado muchos trabajos que evidencian la efectividad de esta alternativa para mejorar los resultados de la carga inmediata en alveolos posextracción.

El objetivo de este caso clínico es demostrar y discutir el tratamiento de un premolar fracturado usando la técnica de socket shield mediante la instalación de un implante inmediato con carga funcional.

\section{REPORTE DE CASO}

Paciente de 47 años, sexo femenino, recurrió a la consulta por fractura coronal de la pieza 24 buscando recuperar el diente fracturado con la instalación de un implante. Después del examen clínico, se constató la fractura y el antecedente de un tratamiento de endodoncia en la pieza 24. Seguidamente, se realizó la evaluación tomográfica, donde se encontró dehiscencia de la tabla vestibular compatible con una posición de la raíz sagital clase I (SRP) (7) (Figura 1).

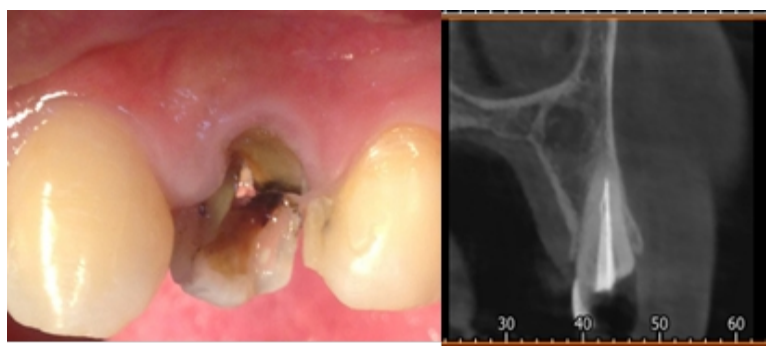

Figura 1: Vista clínica y corte tomográfico de la pza. 24.
Con el fin de preservar la estética anterior, y visto que la raíz no presentaba ninguna lesión apical; la paciente firma un consentimiento informado donde se le explica y detalla el procedimiento de socket shield que se le plantea realizar.

Previa desfocalización y preparación de la paciente, se realizó la cirugía bajo anestesia local. Primero, se eliminó la porción coronal remanente; luego, con una fresa de fisura de tallo largo, se hizo la odontosección y se dividió la raíz en una porción vestibular y otra palatina para proceder a realizar la extracción atraumática de esta última con ayuda de periotomos, dejando la porción vestibular con un espesor entre 1,5 a $2 \mathrm{~mm}$ aproximadamente (Figura 2 ).

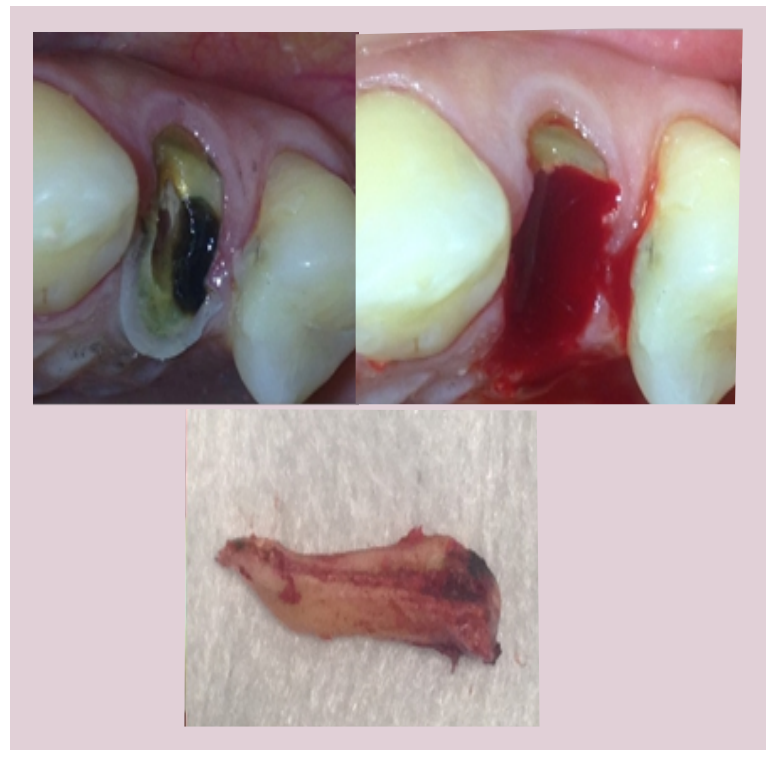

Figura 2: Odontosección para obtener el escudo vestibular y extracción de porción palatina.

Finalmente, con ayuda de una fresa redonda, dejamos el margen coronal del escudo a unos $2 \mathrm{~mm}$ del margen gingival (a nivel de la cresta ósea) con una terminación en chamfer. Se prepará luego el lecho apical del alveolo, aplicando injerto óseo particulado de origen bovino para rellenar el gap. También, se instaló un implante cónico de conexión interna de $3,75 \times 13 \mathrm{~mm}$, obteniendo una buena estabilidad primaria. Inmediatamente, se realizó la impresión a cubeta abierta y se dejó el implante con un tornillo de cicatrización (Figura 3). 


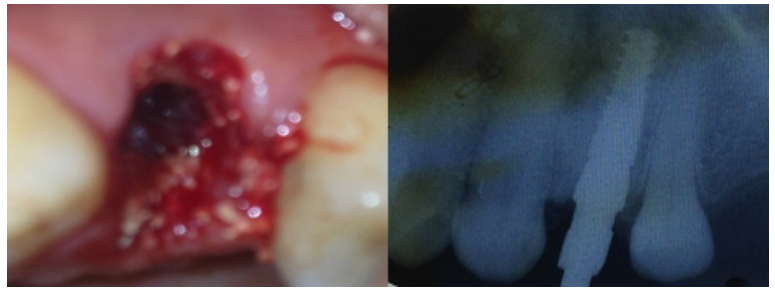

Figura 3: Relleno óseo del alveolo, instalación del implante e impresión a cubeta abierta.

A las 48 horas poscirugía, se instaló una corona definitiva de metal cerámica atornillada. La corona fué controlada dos veces. La primera al mes y medio del tratamiento; y la segunda, un año después. De esta forma, se evidenció la conservación de los tejidos duros y blandos del diente tratado. También, se observó un óptimo resultado funcional y estético.

\section{DISCUSIÓN}

Se ha determinado que la técnica de socket shield parece ser una opción de tratamiento viable para estabilizar tanto la arquitectura gingival vestibular, así comola ósea (8).

Bäumer y col. (9) realizaron un estudio que se centró en la observación histológica, clínica y volumétrica de la cresta alveolar y del implante. Los autores encontraron que el ligamento periodontal se mantiene sano en la porción de la raíz. Observaron también que existe un cambio volumétrico menor del contorno de la cresta y del contacto directo del hueso con el implante. Por tanto, consideraron que esta técnica es una opción fiable.

Roe y col. (8) describen un abordaje quirúrgico diseñado para facilitar la preparación del fragmento de la raíz vestibular, cuando se tiene una posición de la raíz sagital clase I; elevando una ventana vestibular y regenerando la zona fenestrada. A diferencia de ese procedimiento, en este caso clínico, se prefiere dejar esa zona cubierta con biomaterial colocado directamente en el alveolo antes de instalar el implante a fin de evitar realizar un colgajo adicional a la instalación del implante.

Kumar y col. (10) manifiestan que existen ciertas limitaciones en esta técnica. Por ejemplo, la capacitación y preparación del clínico, el procedimiento demanda más tiempo y paciencia para evitar la movilidad del escudo; ya que si el escudo está móvil durante la cirugía, este se debe retirar y realizar la colocación del implante en forma convencional.

La técnica no se recomienda en dientes móviles, dientes que están fuera del arco y dientes con grandes lesiones periapicales. En esta investigación, se concluye que la integridad del escudo juega un papel importante en el éxito del tratamiento.

Bramanti y col. (11) reportaron una tasa de supervivencia del implante del $100 \%$ en sus dos grupos de estudio a los 3 años. Sin embargo, los implantes insertados con la técnica de socket shield mostraron mejores valores tanto para el nivel del hueso marginal, como para la estética gingival $(P<0,05)$. Del mismo modo, Gluckman y col. (12) reportaron una tasa de supervivencia de $96,1 \%$ con un seguimiento entre 1 a 4 años después de la restauración, y una tasa de complicaciones combinadas de un $19,5 \%$, lo cual indica que 5 implantes no se pudieron oseointegrar.

Un espesor mínimo de $2 \mathrm{~mm}$ del escudo en el sitio quirúrgico parece representar uno de los elementos clave para lograr una restauración exitosa (11), coincidiendo con el espesor del escudo que se obtuvo en el manejó en este caso reportado.

Otro punto a tomar en cuenta, es que se debe reducir el escudo al nivel de la cresta ósea, creando un chaflan a 2 $\mathrm{mm}$ de la cresta, adelgazando el escudo ligeramente y proporcionando espacio protésico de 2-3 $\mathrm{mm}$ entre el contorno de la corona subgingival y el escudo para que se dé el relleno de tejidos blandos (12).

En este caso clínico, se usó la técnica de socket shield, donde la tabla vestibular era delgada manteniendo una parte de la raíz vestibular unida al hueso; y la colocación del implante inmediato para mantener el contorno de la cresta y del perfil de emergencia, sin presentar complicaciones posoperatorias. 


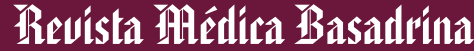

\section{REFERENCIAS BIBLIOGRÁFICAS}

1. Falcón-Guerrero BE. Carga inmediata funcional en la zona estética sobre un implante postextracción. Rev. Mes Periodontol. 2015; 6 (1): 5-9.

2. Falcón GBE. Carga inmediata funcional en la zona estética sobre un implante postextracción: estudio clínico prospectivo. Rev. Mex. Periodontol. 2017; 6 (3): 77-83.

3. Al-Shabeeb MS, Al-Askar M, Al-Rasheed A, Babay N, Javed F, Wang $\mathrm{HL}$ et al. Alveolar bone remodeling around immediate implants placed in accordance with the extraction socket classification: a threedimensional microcomputed tomography analysis. J Periodontol. 2012; 83: 981-87.

4. Kan JYK, Rungcharassaeng K, Deflorian M, Weinstein T, Wang, HL, Testori T. (2018). Immediate implant placement and provisionalization of maxillary anterior single implants. Periodontology. 2000, 77(1), 197-212.

5. Salama M, Ishikawa T, Salama H, Funato A, Garber D. Advantages of the root submergence technique for pontic site development in esthetic implant therapy. Int. j Period Resto Dent. 2007 Dec 1; 27(6):521.

6. Hürzeler MB, Zuhr O, Schupbach P, Rebele SF, Emmanouilidis N, Fickl S. The socket-shield technique: a proof-of-principle report. J clin periodontol. 2010 Sep 1;37(9):855-62.
7. Kan JY, Roe P, Rungcharassaeng K, Patel RD, Waki T. Lozada JL, Zimmweman G. Classification of sagital root position in relation to the anterior maxiliary osseous housing for inmediate implant placement: a cone beam computed tomography study. Int J Oral Maxillofac Implants 2011; 26:873-76.

8. Roe P, Kan JYK, Rungcharassaeng K. Residual root preparation for socket-shield procedures: a facial window approach. Int J Esthet Dent 2017; 12(3):324-335.

9. Bäumer D, Zuhr O, Rebele S, Schneider D, Schupbach P, Hürzeler M. The socket-shield technique: histological, clinical, and volumetrical observations after separation of the buccal tooth segment - a pilot study. Clin Implant Dent Relat Res 2015; 17: 71- 82.

10. Kumar PR, Kher U. Shield the socket: Procedure, case report and classification. J Indian Soc Periodontol 2018; 22:266-72.

11. Bramanti E, Norcia A, Cicciù M, Matacena G, Cervino G, et al. Postextraction Dental Implant in the Aesthetic Zone, Socket Shield Technique Versus Conventional Protocol. Journal of Craniofacial Surgery 2018, 29(4), 1037-41.

12. Gluckman H, Salama M, Du Toit J. A retrospective evaluation of 128 socket-shield cases in the esthetic zone and posterior sites: Partial extraction therapy with up to 4 years follow-up. Clin Implant Dent Relat Res 2017, 20(2), 122-29.

\section{Correspondencia}

Britto Ebert Falcón Guerrero

E-mail: artedent2000@hotmail.com
Fecha de recepción: 05 de junio de 2018

Fecha de aceptación: 25 de octubre de 2018 